\title{
Aplicativo para sondagem de hipótese de escrita infantil remota
}

\author{
Jaline Mombach ${ }^{*}$ \\ jaline.mombach@discente.ufg.br \\ Instituto de Informática \\ Universidade Federal de Goiás \\ Goiânia, Goiás, Brasil
}

\author{
Fabrizzio Soares ${ }^{\dagger}$ \\ soaresf@sou.edu \\ Computer Science Department \\ Southern Oregon University \\ Ashland, Oregon, EUA
}
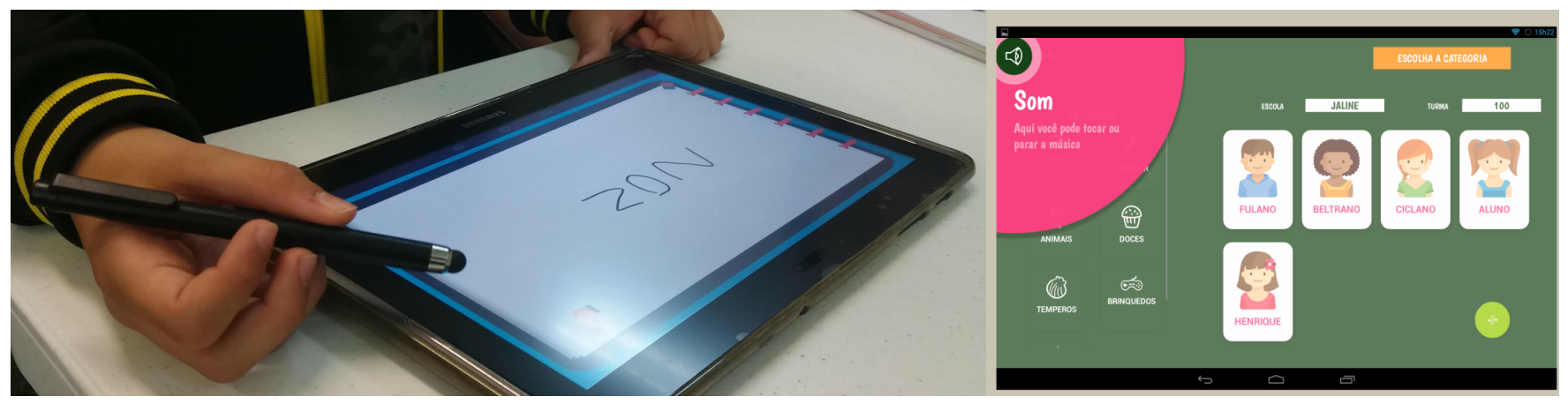

Figura 1: Children Literacy Aid Tool (CLAT).

\begin{abstract}
Nowadays, we face a considerable improvement in education technologies, yet children's spelling assessment is still a paper-andpencil based method. Since it is a regular activity in literacy classrooms and has to be conducted individually with each child, it is a laborious task for a teacher. It is challenging to offer an optimal number of tests because of the number of students in a single class. Moreover, the pandemic period highlighted the need for digital resources to support this activity remotely. In this context, we are developing a method for conducting automated word dictation sessions and support teachers in identifying children spelling development stages. Using DSRM, we believe that we can provide not only offering a more playful writing experience for children through technology, also to reduce teacher overload in the classroom. As preliminary results, the proposal was validated by usability inspection, and the results are promising.
\end{abstract}

\section{KEYWORDS}

Children's Spelling, Educational App, Remote Teaching

\footnotetext{
${ }^{*}$ Doutoranda em Ciência da Computação na Universidade Federal de Goiás. Docente de Informática no Instituto Federal Farroupilha. Lattes: http://lattes.cnpq.br/ 5793944765294091

${ }^{\dagger}$ Chefe do Departamento de Ciência da Computação (Program Chair) na Southern Oregon University e Professor Adjunto (em afastamento) da Universidade Federal de Goiás no Instituto de Informática. Lattes: http://lattes.cnpq.br/7206645857721831
}

In: XIX Workshop de Ferramentas e Aplicações (WFA 2020), São Luís, Brasil. Anais Estendidos do Simpósio Brasileiro de Sistemas Multimídia e Web (WebMedia). Porto Alegre: Sociedade Brasileira de Computação, 2020.

(c) 2020 SBC - Sociedade Brasileira de Computação.

ISSN 2596-1683

\section{INTRODUÇão}

O Plano Nacional de Educação (PNE) determinou diretrizes, metas e estratégias para a política educacional no período de 2014 a 2024 [2]. A meta 5 trata sobre alfabetização e uma das estratégias descritas é fomentar o desenvolvimento de tecnologias educacionais que favoreçam a melhoria do fluxo escolar e a aprendizagem dos alunos. Para atender a essa meta, criou-se o Pacto Nacional pela Alfabetização na Idade Certa (PNAIC), que foi um conjunto de ações firmadas pelo governo federal, estados e municípios.

As prefeituras que aderiram ao programa eram responsáveis pelo monitoramento das avaliações contínuas em sala de aula, conduzidas pelos professores alfabetizadores. Dessas, um número expressivo de secretarias municipais de educação optou por tabelas de acompanhamento de níveis de escrita. Logo, os professores realizavam sondagens de hipótese de escrita de cada criança e preenchiam manualmente planilhas para encaminhamento às prefeituras.

Embora o PNAIC tenha sido descontinuado e substituído recentemente pela Política Nacional de Alfabetização (PNA) [3], a sondagem de hipóteses de escrita é uma atividade rotineira em salas de aula de alfabetização. Inclusive, no Programa Mais Alfabetização, instituído pela Portaria No 142/2018 do Ministério da Educação (MEC), realizam-se sondagens para acompanhamento sistemático das crianças. Essa avaliação usualmente é realizada por meio de ditado de palavras usando lápis e papel [1,4]. Especialistas indicam que o ideal é ter uma visão mais abrangente sobre o registro da criança, não apenas a escrita. É importante que o professor realize a avaliação individualmente e observe sinais estratégicos, como a fala privada ${ }^{1}$, os fonemas pronunciados e o gesto para leitura que a criança faz quando finaliza a escrita.

\footnotetext{
${ }^{1}$ A fala privada é o diálogo que a criança promove consigo mesmo e está diretamente ligada ao processo de aprendizagem.
} 
Identificar qual a hipótese de escrita da criança pode ajudar o professor a criar estratégias de ensino mais eficazes, considerando atividades específicas para o nível do aluno. Entretanto, a pandemia de COVID-19 evidenciou a limitação do processo manual para sondagens. Realizamos uma pesquisa com profissionais de alfabetização e, entre as perguntas, questionamos como eles estavam avaliando a escrita das crianças de maneira remota. Um número expressivo de profissionais indicou dificuldades para essa atividade. A maioria mencionou que não estava realizando sondagens de escrita, outros indicaram que os pais e/ou responsáveis encaminhavam foto do registro das crianças por aplicativos de mensagens instantâneas. Também, recebemos alguns relatos de professores descrevendo a interferência dos familiares durante a escrita dos alunos, dificultando a avaliação real do pensamento da criança.

As ferramentas digitais existentes para ditados, em sua maioria, não satisfazem as especificidades da sondagem de hipótese de escrita infantil. Em nossas pesquisas, identificamos que os aplicativos existentes usam a captura por teclado ou exibem letras para que as crianças as escolham [9]. Porém, essas abordagens podem influenciar na avaliação. Por exemplo, crianças em níveis iniciais podem "escrever" usando símbolos, linhas ou até mesmo desenhos, mostrando que não sabem quais as letras do alfabeto. Logo, ao exibir letras, seja em teclado ou na tela para escolha, o aplicativo conduz esse registro. Ademais, a direção da escrita, o traçado das letras e até mesmo a presença de letras espelhadas ou rotacionadas, são características importantes para a avaliação do professor. Outro aspecto a se destacar é a falta de registro dos demais sinais estratégicos já mencionados, como o toque que a criança faz no papel ao ler o que escreveu e os sons pronunciados durante essa leitura.

O número de crianças em salas de aula de alfabetização dificultam as sondagens individuais inclusive em período regular, com aulas presenciais. Dados da pesquisa mostram que, dependendo do país, a média de alunos nos anos iniciais pode chegar a mais de 30 por turma, como é o caso do Chile. No Brasil, em 2018, a média de alunos foi de 21,6 crianças por turma nos anos iniciais do ensino fundamental [5]. Assim, as avaliações de hipótese de escrita, por exigirem observação individual, demandam significativo esforço do professor e nem sempre conseguem ser realizadas na frequência desejada. Em 2018, o Brasil teve mais de 2,8 milhões de matrículas no $1^{\circ}$ ano do ensino fundamental [6]. Ao considerar que a iniciação à leitura e escrita pode ser realizada na educação infantil e se estender até o $3^{\circ}$ ano do ensino fundamental, há um público expressivo para o desenvolvimento de novas tecnologias voltadas à alfabetização.

Nesse sentido, percebe-se problemas evidentes: (i) Como realizar a sondagem de hipótese de escrita infantil remotamente? (ii) Como possibilitar que as avaliações de hipóteses de escrita infantil sejam massificadas, sem perder as informações individualizadas? (iii) Como facilitar a classificação e análise dessas produções ao professor?

Tendo em vista o cenário exposto, o principal objetivo deste trabalho é desenvolver um método para auxiliar na condução de sessões automatizadas de ditado de palavras para crianças e fornecer suporte à sondagem de hipóteses de escrita infantil aos educadores.

\section{CLAT: DESIGN E DESENVOLVIMENTO}

Inspirando-se nas teorias de desenvolvimento de escrita infantil $[4,12,14]$ e em como os profissionais realizam sondagens de escrita, idealizamos uma tecnologia chamada "Children Literacy Aid Tool"(CLAT). Diante da perspectiva de resolver um problema real por meio de um sistema computacional, adotamos o Design Science Research Methodology (DSRM) [11] como metodologia para delinear a pesquisa. As fases típicas da abordagem de DSRM são sintetizadas na Figura 2. O detalhamento de cada fase do DSRM, no contexto do trabalho, é apresentado em Mombach et al. (2020a)[9].

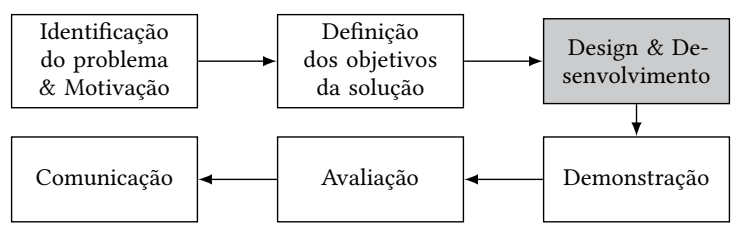

Figura 2: Processo de Design baseado em Peffers et al. [11].

Neste artigo, o foco está na descrição do artefato CLAT. As atividades em $U X$ Research incluíram entrevistas contextuais com professores de alfabetização infantil e também uma pesquisa online para responsáveis e profissionais. Após considerar os requisitos, iniciamos a prototipação e validação. Um protótipo de alta fidelidade foi construído e avaliado por inspeção de usabilidade com especialistas em Interação Humano-Computador (IHC).

\subsection{Arquitetura}

Para desenvolver um método baseado na prática dos professores em sala de aula, dividimos nossa abordagem em três eixos: (i) aquisição, (ii) processamento e (iii) visualização, conforme apresentados na Figura 3.

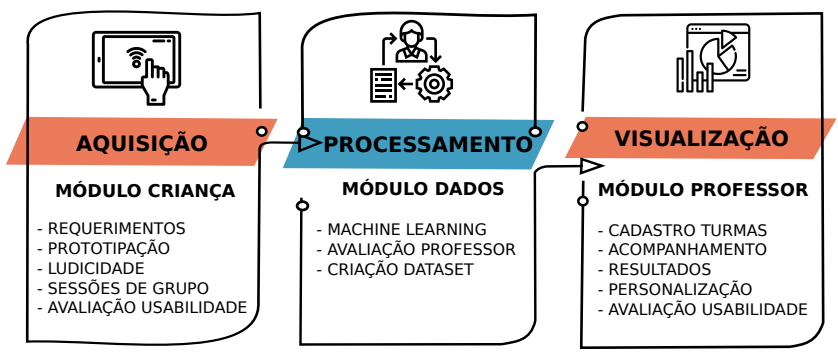

Figura 3: Etapas principais: (i) aquisição; (ii) processamento e (iii) visualização.

2.1.1 Aquisição. A etapa de aquisição tem o objetivo de obter a escrita manual da criança. Para fazer isso, simula-se a ação do professor ditando palavras. Além do registro de escrita, captamos também os sinais estratégicos que o profissional observa nas avaliações presenciais. Dessa maneira, em uma interface lúdica e com interação por voz, desejamos que a criança seja capaz de realizar a sondagem configurada por seu professor ou outro profissional, de forma independente - interagindo apenas com o aplicativo. 
2.1.2 Processamento. Depois de criarmos o aplicativo para a aquisição da escrita da criança, precisamos processar os dados. Atualmente, a aplicação salva os dados da sondagem e cria um relatório similar ao que o professor registra durante a sondagem presencial. Um exemplo desse relatório é apresentado na Figura 4.

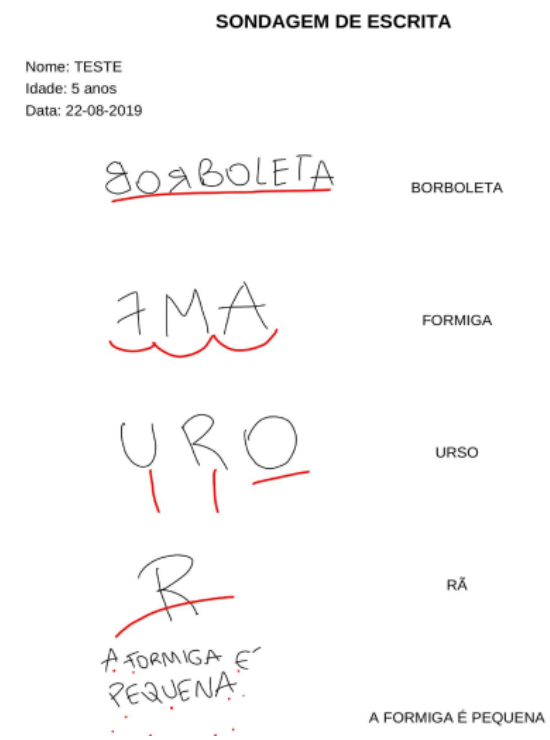

Figura 4: Exemplo de relatório gerado: escrita manual da criança ao lado da palavra/frase que era esperado e linhas vermelhas, representando o movimento de leitura com o dedo na tela.

Apesar do relatório gerado ser exatamente como o professor registra durante a sessão de sondagem, pretendemos gravar também a voz da criança enquanto ela lê. Assim, o profissional poderá rever o movimento realizado e também os sons pronunciados.

Ao criarmos uma base de dados com a coleta de escrita manual das crianças, treinaremos algoritmos de aprendizado de máquina para auxiliar os profissionais em uma avaliação semi-automática. A partir das classificações dos professores, almejamos que o algoritmo aprenda por reforço quais os grupos a serem classificados. Assim, no painel de visualização, mostrará uma pré-classificação da sondagem para que o profissional confirme ou corrija, caso necessário.

Para criar a base de dados temos a parceria do Centro de Ensino e Pesquisa Aplicada à Educação (CEPAE), da Universidade Federal de Goiás para coletar escritas infantis. As imagens manuscritas serão carregadas em um banco de dados na nuvem. O objetivo é criar um conjunto de dados de escrita de crianças público, pois a maioria das bases existentes é privada. Durante a sondagem de hipóteses de escrita das crianças, pediremos aos professores que rotulem o estágio de cada amostra para que possamos auxiliar futuramente na classificação semi-automática.

2.1.3 Visualização. Essa interface funciona como dashboard para o profissional de alfabetização. Relatórios mostram a escrita a mão da criança para cada palavra ditada, o tempo necessário para escrever cada palavra e uma pré-avaliação de qual estágio a criança se qualifica. Além disso, o professor pode ouvir a voz da criança lendo, junto a uma animação que representa o momento em que ela toca na tela durante a leitura. Dessa forma, se a pré-avaliação dada pela algoritmo estiver errada, o professor poderá corrigi-la e essa amostra se tornará parte da base de treinamento da proposta para melhorar a eficácia. A interface de visualização tem também funções para configuração das sessões, ou seja, quantas e quais palavras serão ditadas para as crianças.

Inicialmente, planejamos o módulo de visualização considerando apenas os profissionais de alfabetização. Porém, o período de pandemia enfatizou a necessidade de um modo de visualização para os familiares das crianças também. Dessa maneira, os responsáveis poderão acompanhar o progresso da criança por essa interface.

\section{RESULTADOS}

O projeto CLAT está em desenvolvimento, porém já alcançamos alguns resultados preliminares. Conforme citado anteriormente, algumas etapas do projeto, como a validação da ideia com professores alfabetizadores [10], etapas do desenvolvimento do artefato baseado em DSRM [9], abordagem para reconhecimento e quantificação de letras espelhadas e rotacionadas na escrita manual infantil [7] e a validação de protótipo de alta fidelidade por inspeção de usabilidade [8] já foram realizadas e compartilhadas com a comunidade acadêmica em conferências internacionais.

Além dos artigos científicos, a síntese dos resultados da pesquisa realizada com pais ou responsáveis de crianças de 4 a 7 anos e com profissionais de alfabetização está disponível no site do projeto: https://fabrizziosoares.me/clat. Assim, nesta Seção detalhamos o design experimental e validação do protótipo de alta fidelidade. Entretanto, maiores informações podem ser encontradas nas fontes citadas anteriormente.

\subsection{Design experimental}

O protótipo foi desenvolvido com linguagem Java para Android e usa recursos de armazenamento na nuvem do Firebase ${ }^{2}$. A interface gráfica é baseada nos aplicativos avaliados previamente, incluindo a área de escrita livre. Atualmente, o relatório prestado ao professor é igual ao do papel, em que ele faz marcações sobre como a criança leu.

A Figura 5 mostra o passo a passo do protótipo hi-fi criado. Salientamos que esse protótipo tem funcionalidades limitadas, visto que a função dele era apenas testar a interação com crianças. Logo, pretende-se alterar a proposta para acesso por navegadores convencionais, permitindo uso em diferentes dispositivos sensíveis ao toque, até mesmo com mesas de desenho. O protótipo inicial segue a abordagem de Ferreiro et al. [4], que foi a teoria de desenvolvimento infantil que teve maior destaque na pesquisa que realizamos com professores alfabetizadores.

Nessa abordagem são ditadas quatro palavras de um mesmo campo semântico, que se diferenciam no número de sílabas: polissílaba, trissílaba, dissílaba e monossílaba. Por fim, dita-se uma frase usando uma das palavras anteriores.

No protótipo, o profissional escolhe um dos temas disponíveis e preenche os campos nome da criança e a idade dela. Após, passa o dispositivo para a criança que realizará a sondagem. O aplicativo dita (por text-to-speech) a palavra. Na sequência, a criança escreve

\footnotetext{
${ }^{2}$ https://firebase.google.com
} 

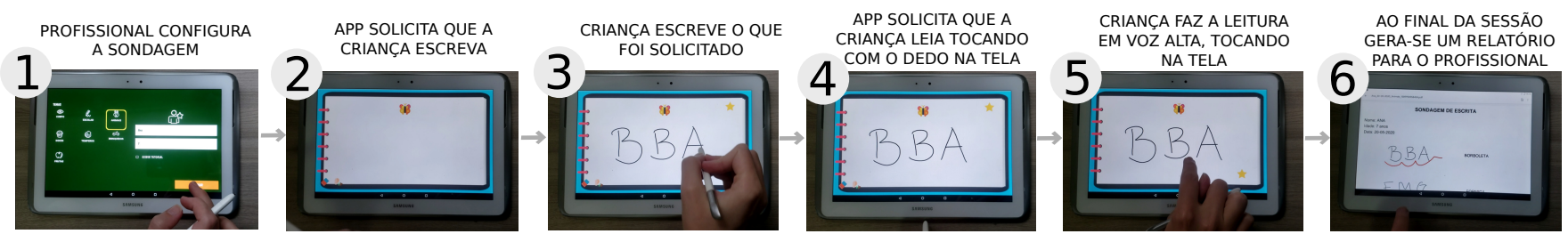

Figura 5: Passo a passo da interação realizada no protótipo de alta fidelidade.

usando o próprio dedo ou uma caneta para dispositivos sensíveis ao toque. $\mathrm{O}$ aplicativo percebe que a criança terminou a escrita e solicita que ela leia em voz alta, tocando com o dedo na tela. $\mathrm{O}$ processo se repete até que todas as palavras e a frase tenham sido ditadas. No final, CLAT gera o relatório para o profissional em formato PDF.

\subsection{Validação}

A pandemia de COVID-19 não permitiu testes de interação com crianças, já que as escolas cancelaram atividades presenciais. Dessa forma, fizemos uma validação preliminar do protótipo com especialistas de Interação Humano-Computador, por meio de inspeção de usabilidade [9].

Para avaliação de usabilidade, escolheu-se as diretrizes do TIDRC [13], um framework conceitual de recomendações de design de interação sensível ao toque para crianças. Nesse framework há 57 recomendações, divididas em três dimensões: socioemocional, física e cognitiva. Comparando a avaliação do protótipo com os resultados obtidos no estudo de Soni et al. [13], percebemos o quão promissora é a proposta CLAT. Embora os aplicativos analisados no estudo não fossem especificamente para alfabetização, a falta de aderência às recomendações de design era crucial.

A pesquisa de Soni et al. [13] relatou que os apps corresponderam apenas a $51 \%$ dos itens na dimensão cognitiva e $67 \%$ na dimensão física. Ao mesmo tempo, CLAT adere até $88 \%$ dos itens na dimensão cognitiva e $75 \%$ na dimensão física. Quanto à dimensão socioemocional, o estudo indica que, em média, $72 \%$ dos aplicativos aderem a esses itens. No CLAT, esses itens precisam ser aprimorados, já que o aplicativo adere apenas $47 \%$ dos itens socioemocionais. Porém, CLAT discorda de alguns itens do TIDRC devido a requisitos definidos pelos professores, principalmente na dimensão socioemocional. Portanto, fica evidente a ampliação de diretrizes específicas para aplicativos infantis sensíveis ao toque no que diz respeito à alfabetização precoce, incluindo recursos pedagógicos.

Os resultados alcançados até o presente momento mostram que a proposta é relevante e viável, possibilitando que seja dada continuidade à investigação.

\subsection{Análise de impacto}

A demanda desse projeto surgiu a partir dos professores de alfabetização. Espera-se como principal mudança a diminuição de tempo para aplicação de sondagens de escrita infantil e possibilidade de uso remoto.
Realizamos uma pesquisa com profissionais ${ }^{3}$ e questionamos quanto tempo, em média, é utilizado para aplicação de sondagem de escrita. A maioria dos participantes indicou até 30 minutos por aluno. Logo, em uma turma com 20 alunos, seria necessário 600 minutos (10 horas) dedicadas exclusivamente a esse atendimento individual. Na prática, os professores relatam que precisam diluir as avaliações em várias semanas, aplicando sondagens com apenas 2 a 3 alunos por dia. Além disso, enquanto o professor está realizando a sondagem é necessário que outro adulto fique responsável pelos demais alunos da turma.

Com a nossa proposta, as sondagens de avaliação da escrita poderão ser aplicadas simultaneamente a todos os estudantes. Dessa maneira, prevemos diminuição de tempo de aplicação de até 570 minutos, quando comparado ao método atual. Assim, o professor poderá aplicar mais vezes a sondagem de hipótese de escrita em suas turmas, o que facilita o planejamento personalizado às necessidades de cada criança, conforme a sua fase ou nível de escrita. Em larga escala, pode contribuir indiretamente na melhoria dos índices de alfabetização brasileiros.

Como investimentos, nossa abordagem necessita de dispositivos sensíveis ao toque. Porém, o investimento nesse tipo de dispositivo não é exclusivo para a proposta, ou seja, os professores poderiam utilizar também para outros fins pedagógicos. Na impossibilidade de investimento em dispositivos específicos, como tablets, é possível utilizar em smartphones pessoais, exatamente como a maioria da população brasileira está aderindo ao ensino remoto no momento, por meio de smartphones próprios ou de familiares das crianças.

Quanto à escalabilidade, é possível alterar o idioma e usar em outros países, visto o nível de personalização permitido ao professor. Por fim, a solução será distribuída em licença open source e com uso gratuito por escolas e instituições de atendimento infantil públicas.

\subsection{Resultados esperados}

Após a conclusão deste trabalho, espera-se que seja possível construir um modelo para sondagem de escrita remota, que modernize o método atual de avaliação e possa facilitar a intervenção do educador na realização da sondagem. Consequentemente, após o período de pandemia, será possível realizar testes com maior frequência nas turmas de alfabetização. O professor poderá usar os modelos já fornecidos pela aplicação ou personalizar as palavras conforme a sua preferência ou seguindo o tema que esteja explorando em sala de aula. Além disso, o método com avaliação semi-automática poderá tornar-se um objeto de aprendizagem para professores em

${ }^{3}$ Pesquisa com profissionais disponível em https://fabrizziosoares.me/clat/resultado_
professores.html 
formação, que estudam a tese e precisam aprender a diagnosticar os níveis das crianças.

No ensino remoto, percebemos que a estratégia pode auxiliar na aplicação das sondagens a distância. O período de pandemia evidenciou essa necessidade, porém há diversos contextos em que o ensino remoto é demandado. Por exemplo, crianças em fase de alfabetização que estejam hospitalizadas ou em tratamento de alguma doença em casa. O recurso também possibilita que o professor encaminhe avaliações extras, como "tema de casa"e compartilhe facilmente os resultados com demais profissionais que atendam a criança, como psicólogos, fonoaudiólogos, neurologistas e outros.

Também, pretende-se oferecer uma experiência de escrita avançada para a criança, reproduzindo a experiência do papel e lápis adaptada em diferentes dispositivos sensíveis ao toque e promover maior engajamento do aprendiz durante as sessões de escrita por meio de estratégias de gamificação.

\subsection{Cronograma}

Os passos seguintes serão essenciais para testar se a hipótese apresentada é válida. Logo, os trabalhos futuros indicam teste do protótipo com as crianças, estudo e implementação de técnicas de aprendizado de máquina para que o sistema seja capaz de aprender o método de avaliação do professor e o desenvolvimento do módulo de visualização.

As atividades previstas, após adaptação do cronograma, são:

- 2020/2: implementação de visualização para pais e/ou responsáveis. Adaptação do protótipo para plataforma web.

- 2021/1: captura de escrita e avaliação do protótipo com crianças.

- 2021/2: correções de design e implementação de aprendizado de máquina com a base de dados criada.

- 2022/1: lançamento da plataforma CLAT e período de testes e avaliação com usuários.

As atividades de captura de escrita e avaliação do protótipo com crianças e professores estava prevista para o primeiro semestre de 2020. No entanto, devido ao período de pandemia, não foi possível a realização. Logo, o projeto precisou ser adiado devido necessidade de base de dados para continuidade do desenvolvimento e inclusão de algoritmos de aprendizado de máquina.

No momento, estão sendo desenvolvidos os módulos que possam ser antecipados, mesmo sem a avaliação prévia de usuários, a fim de manter o projeto ativo. Nessa perspectiva, o desenvolvimento do dashboard para pais/responsáveis e profissionais. Também, pretende-se adaptar o protótipo para tecnologias web, ampliando o funcionamento para além de dispositivos Android.

As bases existentes são ineficientes para a proposta, visto que o público do CLAT são crianças de 4 a 7 anos, que possuem escrita a mão com características muito específicas, seja pela direção livre, espessura do traço ou pela presença de letras espelhadas e rotacionadas. Essa fase foi planejada para realização presencial. Entretanto, estamos adaptando um módulo para captura de escrita que facilite a aquisição usando smartphones para compor a base de dados.

Devido à pandemia, a avaliação com professores e crianças foi adiado para o ano letivo de 2021, conforme aprovação do Conselho Diretor do CEPAE/UFG pelo calendário escolar da Instituição e pelo cadastro na Plataforma Brasil - Comitê de Ética em Pesquisa.

\section{CONSIDERAÇÕES FINAIS}

Este trabalho propõe o uso de um conjunto de técnicas para que seja possível auxiliar na condução e avaliação semi-automática de hipóteses de escrita infantil. O método aborda três eixos de atuação: aquisição dos manuscritos, processamento automatizado e ainda o módulo de visualização que ajudará o professor na análise e acompanhamento do progresso de seus alunos. Atualmente, o projeto desenvolveu um protótipo $h i-f i$ para o primeiro eixo, em que foi iniciada a exploração de reconhecimento automático de caracteres para os objetivos do segundo eixo.

O protótipo já foi inicialmente avaliado em formato piloto tanto em sessões com professores colaboradores, como por especialistas em IHC, e os resultados observados foram bastante satisfatórios. Desta forma, temos uma expectativa positiva de utilização do método proposto em sala de aula, com potencial para impactar no custo/tempo de aula bem como na qualidade da avaliação.

\section{REFERÊNCIAS}

[1] Claude Beucher-Marsal, Florence Charles, and Carole Le Hénaff. 2015. Improving Literacy Skills and Differentiating Learning Speed among Primary School Children through a Computer-Assisted Learning Tool. (2015).

[2] Brasil. 2014. Lei $n^{\circ}$ 13.005/2014 - Aprova o Plano Nacional de Educação - PNE e dá outras providências. Diario Oficial [da] Republica Federativa do Brasil (jun 2014). http://www.planalto.gov.br/ccivil_03/_ato2011-2014/2014/lei/l13005.htm

[3] Brasil. 2019. Decreto $\mathrm{n}^{\circ} 9.765$ - Institui a Política Nacional de Alfabetização. Diario Oficial [da] Republica Federativa do Brasil (11 abr. 2019). http://www.in.gov.br/materia/-/asset_publisher/Kujrw0TZC2Mb/content/id/ 71137476/do1e-2019-04-11-decreto-n-9-765-de-11-de-abril-de-2019-71137431

[4] Emília Ferreiro, Ana Teberosky, and Diana Myriam Lichtenstein. 1986. Psicogênese da língua escrita. Artes Médicas.

[5] INEP. 2018. Indicadores Educacionais. http://portal.inep.gov.br/indicadoreseducacionais

[6] INEP. 2019. Sinopse Estatística da Educação Básica 2018. http://portal.inep.gov. $\mathrm{br} / \mathrm{web} /$ guest/sinopses-estatisticas-da-educacao-basica

[7] Jaline Mombach, Cristiane Ferreira, Juliana Felix, Rogerio Salvini, and Fabrizzio Soares. 3002 in press 2020. Remote Assessing Children's Handwriting Spelling on Mobile Devices. In 2020 IEEE Canadian Conference of Electrical and Computer Engineering (CCECE). IEEE.

[8] Jaline Mombach, Afonso Fonseca, Thamer Nascimento, Welington Rodrigues, Henrique Gressler, Fábio Diniz Rossi, , and Fabrizzio Soares. 2020. A new approach to performing paper-based children's spelling tests on mobile devices. In 2020 IEEE International Conference on Systems, Man and Cybernetics (SMC). IEEE.

[9] Jaline Mombach, Fábio Diniz Rossi, Juliana Felix, and Fabrizzio Soares. 2020. Remote Assessing Children's Handwriting Spelling on Mobile Devices. In 2020 IEEE 44th Annual Computers, Software, and Applications Conference (COMPSAC). IEEE, 1279-1284

[10] Jaline Mombach, Paulo Silas Souza, Fabrizzio Alves de Melo Nunes Soares, Fabio Rossi, Tiago Ferreto, and Lara Leal. 2019. An approach for children's emergent literacy adaptive activities. In Brazilian Symposium on Computers in Education (Simpósio Brasileiro de Informática na Educação-SBIE), Vol. 30. 489.

[11] Ken Peffers, Tuure Tuunanen, Marcus A Rothenberger, and Samir Chatterjee. 2007. A design science research methodology for information systems research. Journal of management information systems 24, 3 (2007), 45-77.

[12] Tatiana Cury Pollo, Rebecca Treiman, and Brett Kessler. 2007. Three perspectives on spelling development. In Single-Word Reading. Psychology Press, 187-201.

[13] Nikita Soni, Aishat Aloba, Kristen S Morga, Pamela J Wisniewski, and Lisa Anthony. 2019. A Framework of Touchscreen Interaction Design Recommendations for Children (TIDRC) Characterizing the Gap between Research Evidence and Design Practice. In Proceedings of the 18th ACM International Conference on Interaction Design and Children. 419-431.

[14] Rebecca Treiman, Brett Kessler, Tatiana Cury Pollo, Brian Byrne, and Richard K Olson. 2016. Measures of kindergarten spelling and their relations to later spelling performance. Scientific Studies of Reading 20, 5 (2016), 349-362. 